Artículo

\title{
Fermentación ruminal y producción de metano usando la técnica de gas in vitro en forrajes de un sistema silvopastoril de ovinos de Chiapas, México
}

Ángel Jiménez-Santiago ${ }^{\mathrm{a}}$

Guillermo Jiménez-Ferrer ${ }^{\text {a* }}$

Armando Alayón-Gamboa ${ }^{\text {b }}$

Esaú de Jesús Pérez-Lunac

Ángel Trinidad Piñeiro-Vázquez ${ }^{\mathrm{d}}$

Samuel Albores-Moreno ${ }^{\mathrm{b}}$

Ma. Guadalupe Pérez-Escobar ${ }^{\mathrm{a}}$

Ricardo Castro-Chan ${ }^{\mathrm{d}}$

${ }^{a}$ ECOSUR (El Colegio de la Frontera Sur, Unidad SCLC), Departamento de Agricultura, Sociedad y Ambiente. Carr. Panamericana s/n, San Cristóbal de las Casas, Chiapas, México.

${ }^{\mathrm{b}}$ ECOSUR (Unidad Campeche). Campeche, México.

c UNACH (Universidad Autónoma de Chiapas), Facultad de Agronomía. Chiapas, México.

d Tecnológico Nacional de México. IT Conkal. Yucatán, México.

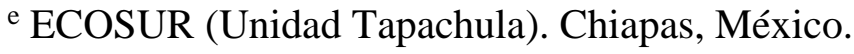

* Autor para correspondencia: gjimenez@ecosur.mx 


\section{Resumen:}

Se evaluaron mediante la técnica de producción de gas in vitro, fuentes energéticas locales (melaza, Zea mays L. y Musa paradisiaca L.) sobre la fermentación ruminal y producción de metano de diversos forrajes usados en un sistema silvopastoril con Panicum maximum cv. Tanzania, Gliricidia sepium (Jacq.) y Leucaena leucocephala cv. Cunningham, con ovinos. Se usaron cinco borregos Pelibuey x Katahdin $40 \pm 3(\mu \pm \mathrm{DE}) \mathrm{kg}$ como donantes de líquido ruminal. Se analizaron cinco tratamientos (dietas) con diferentes mezclas de follaje de arbóreas y fuentes energéticas en un diseño experimental completamente al azar. M. paradisiaca y Z. mays presentaron los mayores registros de volumen (V) máximo en producción de gas ( 544 y $467 \mathrm{ml} / \mathrm{g}^{-1} \mathrm{MS}$, respectivamente) $(P \leq 0.05)$. El follaje de G. sepium y L. leucocephala tuvieron los menores valores de V (253 y $180 \mathrm{ml} / \mathrm{g}^{-1} \mathrm{MS}$, respectivamente) $(P \leq 0.05)$. La dieta D4 GMP (48 \% P. maximum, $30 \%$ G. sepium, $7 \%$ Zea mays, $15 \%$ M. paradisiaca) registro el mayor valor de V. No hubo diferencia $(P>0.05)$ en la producción de metano en las dietas usadas, teniendo un rango de 6.31 a 9.60 de LCH4/kg MSDIG. Se generó un índice de emisión potencial de gases fermentables (IPEGF), el cual sugirió que dietas con carbohidratos de lenta fermentación, contribuyen a un índice más alto de emisión de gases. Por su mejoramiento en la calidad de las dietas y en contribuir en una baja de emisiones de $\mathrm{CH}_{4}$, se sugiere el manejo de arbóreas forrajeras como G. sepium y L. leucocephala, incorporando fuentes energéticas locales.

Palabras clave: Mitigación, Cambio climático, Energía, Agroforesteria.

Recibido: 13/06/2017

Aceptado: 29/05/2018

\section{Introducción}

Se ha reconocido que la ganadería tiene un papel fundamental en la sobrevivencia de más de 800 millones de pobres en el mundo ${ }^{(1)}$. Sin embargo, la producción animal también contribuye en la degradación de los recursos naturales, la contaminación ambiental y el cambio climático $(\mathrm{CC})^{(2)}$, principalmente por su contribución en las emisiones de gases de efecto invernadero $(\mathrm{GEI})^{(3)}$. La ganadería tropical en América Latina, primordialmente está basada en el pastoreo de pastos naturales e introducidos bajo sistemas extensivos ${ }^{(4)}$, con escasa o nula suplementación, escasa infraestructura y poco capital ${ }^{(5)}$. En este contexto, los sistemas silvopastoriles y el aprovechamiento de árboles y arbustos forrajeros locales, ha sido una opción viable para mejorar los sistemas productivos ganaderos, reducir su impacto ambiental y contribuir en la mitigación de los GEI ${ }^{(6-9)}$. 
En sistemas silvopastoriles, la presencia de proteína en el follaje de árboles de uso múltiple en los géneros botánicos de Leucaena, Gliricidia, Erythrina, entre otros, es rápidamente degradable en el rumen, lo que hace necesario la incorporación de alimentos energéticos para mejorar la eficiencia de la fermentación, sincronía y balance de nutrientes en el rumen ${ }^{(10,11)}$. Por otra parte, los subproductos energéticos comerciales de alta calidad para los sistemas ganaderos que producen carne o leche, tienen un alto costo monetario $^{(12)}$, por lo cual se requiere buscar suplementos basados en recursos locales de fácil acceso y aceptable valor nutritivo ${ }^{(13)}$. Se sabe que en el follaje de muchos árboles forrajeros hay presencia de metabolitos secundarios ${ }^{(14)}$, los cuales tienen la capacidad de mitigar las emisiones de metano entérico en los rumiantes ${ }^{(15,16)}$. Así, hay evidencias que indican que el follaje de árboles forrajeros tienen la capacidad de reducir la población de protozoarios y archaeas metanogénicas ${ }^{(17,18,19)}$, provocando una menor síntesis $\mathrm{y}$ producción de $\mathrm{CH}_{4}$ entérico ${ }^{(20)}$.

El objetivo de este estudio fue evaluar el efecto de diversas fuentes energéticas locales sobre los parámetros de fermentación ruminal y emisiones de metano usando forrajes de un sistema silvopastoril de ovinos en pastoreo ( $P$. maximum) complementados con follaje de Gliricidia sepium y Leucaena leucocephala.

\section{Material y métodos}

\section{Área de estudio}

Los materiales para desarrollar este estudio se obtuvieron de un rancho productor de ovinos con manejo silvopastoril en el municipio de Chiapa de Corzo, Chiapas, México $\left(16^{\circ} 42^{\prime} \mathrm{N}\right.$ y $\left.93^{\circ} 00^{\prime} \mathrm{W}\right)$. La unidad ganadera tiene una altitud de 400 a $450 \mathrm{msnm}$, con una precipitación promedio anual de $900 \mathrm{~mm}$ y con temperatura media anual de $26.0^{\circ} \mathrm{C}$. El suelo predominante es de textura franca, con contenido de materia orgánica de $2.4 \%$, $\mathrm{pH}$ de 7.0 y ligeramente pobre en nitrógeno $(0.15 \%)^{(21)}$. La unidad silvopastoril tiene 12 ha y un hato promedio de 55 vientres de ovinos de la raza Pelibuey x Katahadin. Se cuenta con 10 ha para pastoreo con pasto Tanzania (P. máximum) con cercos vivos de $L$. Leucocephala (Guash= nombre comun), G. sepium (Cocoite= nombre común) y Cordia dentata (Vahl) (Nanguipo= nombre común). Diversos potreros (3 ha) también tienen árboles de L. Leucocephala en callejones y 7 ha tienen árboles dispersos en las áreas de pastoreo como Enterolobium cyclocarpum (Jacq) y Ceiba pentandra L. Se cuenta con 2 ha de reserva natural de selva baja caducifolia, no se utiliza fertilización química de pasturas, hay manejo rotacional de potreros mediante cercos eléctricos y uso de riego en épocas de estiaje. El destino de los animales es para el mercado de carne de ovinos regional y nacional. 


\section{Análisis químico de los alimentos usados}

La determinación de materia seca (MS) de los forrajes y suplementos usados se realizó en una estufa de aire forzado a $55^{\circ} \mathrm{C}$ por $48 \mathrm{~h}$ (peso constante) según la NOM-116-SSA11994. El contenido de proteína cruda se realizó mediante un método interno (ECOSURET-BR04) con base en la norma NMX-F-608-NORMEX-2002. El contenido de materia orgánica (MO) se evaluó mediante incineración en una mufla a $550{ }^{\circ} \mathrm{C}$ durante $3 \mathrm{~h}$ según la norma NMX-F-607-NOMRMEX-2002 y el contenido de fibra detergente neutra (FDN) y fibra detergente acida (FDA) se determinó según lo sugerido por Van Soest ${ }^{(22)}$ usando el procedimiento secuencial, con uso de alpha - amilasa y sin corrección de cenizas en todas las muestras (AOAC) ${ }^{(23)}$. Los taninos condensados se determinaron por el método de vainillina acidificada (solución al $1 \% \mathrm{p} / \mathrm{v}$ de vainillina en metanol) ${ }^{(24)}$.

\section{Producción de gas in vitro}

Se realizó un ensayo de gas in vitro utilizando la metodología de la técnica acumulativa de gas sugerida por Theodorou ${ }^{(25)}$ y Williams ${ }^{(26)}$. Se consideraron seis materias primas y se diseñaron cinco dietas (tratamientos) (Cuadro 1): $P$. maximum como forraje base (control), G. sepium y L. leucocephala como fuentes de proteína. Melaza, Z. mays y M. paradisiaca (guineo verde) como fuentes energéticas. La formulación de las dietas fueron isoenergeticas e isoproteicas formuladas para cubrir las demandas de ovinos adultos utilizados en la unidad silvopastoril, con un contenido de 2,200 kcal/kg y $14 \%$ de PC. La fermentación in vitro de cada uno de los tratamientos se realizó por triplicado en viales de vidrio color ámbar de $90 \mathrm{ml}$ introduciendo $0.5 \pm 0.001 \mathrm{~g}$ de sustrato, y se evaluó la fermentación mediante la producción de gas a diferentes tiempos $(0,2,4,6,8$, $10,12,16,20,24,30,36,48,60,72$ h). El manejo de los borregos y la extracción del líquido ruminal se realizó de acuerdo a lo indicado por Alexander y McGowan ${ }^{(27)}$, Blummel y Orskov ${ }^{(28)}$ y bajo las normas de bienestar animal del grupo de investigación en ganadería sustentable de ECOSUR. Para la extracción de líquido ruminal se utilizaron cinco borregas del área experimental con un peso vivo de $40.0 \pm 3.0 \mathrm{~kg}$, edades similares y condición corporal de 3.5 promedio. Se extrajeron $300 \mathrm{ml}$ de líquido ruminal por animal por medio de una sonda esofágica para obtener un total $1.5 \mathrm{~L}$. Las muestras se mantuvieron a $39{ }^{\circ} \mathrm{C}$ protegidas de la luz solar. El monitoreo de la presión generada en cada vial se realizó con un manómetro analógico (Marca: METRON Mod.63100 rango: $0-1 \mathrm{~kg} / \mathrm{cm}^{2}$ ), y se generaron las siguientes variables de respuesta: volumen máximo de gas (V), tasa de producción de gas (S), fase de retraso (L), volúmenes fraccionales de gas generados durante el ensayo: $\mathrm{V}_{8}$ : volumen fraccional de rápida fermentación en las primeras 8 h, $V_{24}$ : volumen generado en la fracción media, entre 8-24 h de fermentación, y $V_{72}$ : volumen generado en la fracción entre 24 a 72 h de fermentación. Se realizaron dos tandas de incubación de forma simultánea, cada tanda comprendía tres repeticiones 
(viales) por cada alimento y tratamiento, respectivamente. La primera tanda fue para evaluar la producción de gas total acumulado a 72 h, en cada una de las fracciones fermentables: fracción rápida, fracción media y fracción lenta. En las fracciones fermentables se estimaron tres grupos de carbohidratos fermentables (monosacáridos, almidón y celulosa) de acuerdo a los volúmenes de gas obtenido para los intervalos de tiempo de 0 a $8\left(\mathrm{Vf}_{0-8}\right), 8$ a $24\left(\mathrm{Vf}_{8-24}\right)$ y 24 a $72\left(\mathrm{Vf}_{24-72}\right)$ horas de incubación. Estos volúmenes se utilizaron para estimar de acuerdo con las ecuaciones de regresión lineal propuestas por Miranda et $a l^{(29)}: \mathrm{FR}=\mathrm{Vf}_{0-8} / 0.4266, \quad \mathrm{FM}=\mathrm{Vf}_{8-24} / 0.6152, \quad \mathrm{FL}=\mathrm{Vf}_{24}$ 72/0.3453) las fracciones de rápidamente, medianamente y lentamente fermentables. Los valores de producción de gas acumulado se ajustaron al modelo de Menke and Steingas ${ }^{(30)}$ :

Cuadro 1: Tratamientos y porcentaje de ingredientes usados en el experimento de gas In vitro en Chiapas, México

\begin{tabular}{lcccccc}
\hline Alimentos & P. maximum & G.sepium & L. leucocephala & M. Paradisiaca & Z. mays & Melaza \\
\hline P100 (control) & 100 & 0 & 0 & 0 & 0 & 0 \\
G100 & 0 & 100 & 0 & 0 & 0 & 0 \\
L100 & 0 & 0 & 100 & 0 & 0 & 0 \\
MP100 & 0 & 0 & 0 & 100 & 0 & 0 \\
Z100 & 0 & 0 & 0 & 0 & 100 & 0 \\
M100 & 0 & 0 & 0 & 0 & 0 & 100 \\
Tratamientos & 47 & 0 & 30 & 0 & 8 & 15 \\
D1LM & 47 & 0 & 30 & 15 & 8 & 0 \\
D2LMP & 47 & 30 & 0 & 0 & 8 & 15 \\
D3GM & 48 & 30 & 0 & 17 & 5 & 7 \\
D4GMP & 47 & 16 & 17 & 5 & 0 \\
D5GLMPM & & & & & 10 \\
\hline
\end{tabular}

P100 (control)= P. máximum $; \mathrm{G} 100=$ G.sepium $; \mathrm{L} 100=$ L. leucocephala $; \mathrm{MP100}=$ M.paradisiaca $; \mathrm{Z} 100=Z$. mays $;$ M100= melaza; $\mathrm{D} 1 \mathrm{LM}=47 \%$ P. maximum, $30 \%$ L. Leucocephala, $8 \% \mathrm{Z}$. mays, $15 \%$ melaza; D2LMP $=47 \%$ P. maximum, $30 \%$ L. Leucocephala $8 \%$ Z. mays, $15 \%$ M. paradisiaca; D3GM: $47 \%$ P. maximum, $30 \%$ G. sepium, $8 \%$ Z. mays, $15 \%$ melaza; D4GMP $=48 \%$ P. maximum, $30 \%$ G. sepium., $7 \%$ Zea mays, $15 \%$ M. paradisiaca; D5GLMPM= 47\% P. maximum, $16 \%$ G. sepium $17 \%$ L. leucocephala. $5 \%$ M. paradisiaca., $5 \%$, Z. mays, $10 \%$ melaza.

$$
\mathrm{Y}=\mathrm{v} /(1+\exp (2-4 * \mathrm{~s} *(\mathrm{t}-\mathrm{L})))
$$

Donde:

$\mathbf{Y}=$ volumen total de gas producido

$\mathbf{v}=$ volumen máximo de producción

$\mathbf{s}=$ tasa constante de producción de gas,

$\mathbf{t}=$ tiempo,

$\mathbf{L}=$ fase lag o de retraso.

La digestibilidad in vitro de la materia seca (DIVMS) se obtuvo mediante análisis gravimétricos, tomando en cuenta el peso de la MS inicial y la final obtenida a 24 y $72 \mathrm{~h}$ después de iniciada la fermentación, recuperando el material mediante filtrado (200 
micrómetros) y secado de material a $65^{\circ} \mathrm{C}$ hasta peso constante, utilizando la siguiente fórmula:

$$
\% D I V M S=\frac{P I-P F}{P I} * 100
$$

Donde:

\% DIVMS= porcentaje de digestibilidad in vitro de la materia seca;

$\mathbf{P I}=$ peso inicial de la materia seca incubada en gramos,

$\mathbf{P F}=$ peso final de la materia seca incubada en gramos. Con los datos de DIVMS $24 / 72$ y volúmenes de gases emitidos en la fermentación, se generó un índice potencial de emisión de gases fermentables (IPEGF), Este índice hace referencia a la cantidad de gas que puede producir un sustrato por cada gramo de MS o MO fermentada en el rumen ${ }^{(31)}$.

\section{Determinación de la producción de metano}

Con las muestras de la segunda tanda de incubación se analizó la producción de $\mathrm{CO}_{2}$ y $\mathrm{CH}_{4}$ y gases menores durante las primeras 24 horas de fermentación. Se realizó la separación del $\mathrm{CO}_{2}$ por medio de una trampa (frasco de vidrio herméticamente sellado con tapón de hule y aro de aluminio) que contenía $90 \mathrm{ml}$ de hidróxido de potasio (KOH) a una concentración de uno molar y una dilución de $56.10 \mathrm{~g}$ de $\mathrm{KOH}$ en un litro de agua desionizada de acuerdo a la metodología propuesta por Bartha y Pramer ${ }^{(32)}$ modificada por Miranda ${ }^{(29)}$. Se tomó una muestra en viales estériles y al vacío para su posterior análisis en cromatografía de gases y cuantificar el $\mathrm{CH}_{4}$ por cada sustrato. Para el análisis de la producción de $\mathrm{CH}_{4}$ se utilizó un cromatógrafo de gases marca PERKIN ELMER CLARUS 500, Software Versión 6.3.2.0646, diámetro de columna $0.530 \mathrm{~mm}$ y $50 \mathrm{~m}$ de largo, con una temperatura de inyección de $35^{\circ} \mathrm{C}$. Se analizaron 36 muestras recolectadas durante la fermentación in vitro de 24 h, en la segunda corrida de incubación, inyectando $20 \mu \mathrm{l}$ de muestra por cada análisis. Las concentraciones de $\mathrm{CH}_{4}$ se corrigieron en cada tratamiento restando la producción de metano promedio de los tres blancos. Para calcular la concentración y el efecto de los tratamientos sobre la producción de $\mathrm{CH}_{4}$ se expresó en $\mathrm{L}$ de $\mathrm{CH}_{4} / \mathrm{kg}$ MSDIG.

\section{Análisis estadístico}

Los parámetros de producción de gas, DIVMS y producción de metano se analizaron mediante ANOVA en un diseño completamente al azar. El modelo matématico fue:

$$
Y_{i j}=\mu+T_{i}+\varepsilon_{i j}
$$




\section{Donde:}

$\boldsymbol{Y}_{i j}=$ Variable respuesta en la $j$-ésima repetición (frascos) del $i$-ésimo tratamiento.

$\boldsymbol{\mu}=$ es la media global de todos los datos del experimento.

$T_{i}=$ Efecto del tratamiento $i$.

$\varepsilon_{i j}=$ el error experimental asociado al $j$ sujeto bajo el $i$ tratamiento.

Los datos obtenidos de todas las variables de respuesta fueron sometidos a un análisis de varianza $^{(33)}$. Las diferencias entre promedios de los tratamientos se compararon con la prueba de Tukey $(P \leq 0.05)$ mediante PROC GLM del SAS ${ }^{(34)}$.

\section{Resultados y discusión}

El cuadro 2 muestra la composición química de los forrajes, fuentes energéticas y tratamientos (dietas) usados en el presente experimento. El contenido proteico (PC) en el follaje de G. sepium y L. leucocephala fue alto, siendo superior a lo reportado por otros autores $^{(32,33)}$. Como era de esperarse, las fuentes energéticas tuvieron bajos contenidos de PC y FDN. El pasto P. máximum (control) tuvo una buena concentración de PC si se compara con los valores promedio de pastos tropicales, los cuales se encuentran normalmente entre 7 y $9 \%$ de PC. Esta alta concentración de PC en la pastura puede estar asociado a la fertilización natural derivadas de las excretas de los ovinos bajo un manejo de pastoreo controlado. La pastura presentó altos contenidos de FDN y FDA. Se encontraron escasos taninos condensados (TC) en L. leucocephala, comparado con otros estudios $^{(35,36)}$. Esto pudo deberse a la variabilidad en las características del valor nutricional del follaje en árboles forrajeros de la misma especie, debido a condiciones de sitio, manejo, etapa fenológica y particulares del área de estudio ${ }^{(37)}$. El contenido de lignina en L. leucocephala encontrado fue alto, sin embargo esta dentro de los rangos sugeridos por la FAO. La cantidad de lignina presente en la L. leucocephala de este ensayo, afectó directamente su digestibilidad y seguramente los componentes de la ración, reduciendo el aprovechamiento de energía ${ }^{(38,39)}$. 
Cuadro 2: Composición química (g/Kg MS) de los forrajes, fuentes energéticas y tratamientos usados en el experimento de gas in vitro en Chiapas, México

\begin{tabular}{|c|c|c|c|c|c|c|c|c|}
\hline & MS & MO & PC & Lignina & FDN & FDA & TC & CHO \\
\hline P. maximum (control) & 933 & 853 & 124 & 103 & 712 & 490 & NA & 231 \\
\hline G. sepium & 930 & 889 & 367 & 133 & 353 & 250 & 0 & 269 \\
\hline L. leucocephala & 932 & 883 & 261 & 207 & 462 & 308 & 56 & 352 \\
\hline M. paradisiaca & 925 & 953 & 52 & NA & 137 & 37 & NA & 763 \\
\hline Z. mays & 866 & 984 & 59 & 6 & 86 & 16 & NA & 795 \\
\hline Melaza & 788 & 866 & 53 & $3 *$ & $8^{*}$ & $5^{*}$ & NA & 600 \\
\hline D1LM & 906 & 874 & 149 & 111 & 481 & 324 & 16 & 368 \\
\hline D2LMP & 926 & 887 & 149 & 111 & 501 & 329 & 16 & 392 \\
\hline D3GM & 905 & 876 & 181 & 89 & 448 & 307 & NA & 343 \\
\hline D4GMP & 926 & 888 & 182 & 90 & 474 & 317 & NA & 361 \\
\hline D5GLMPM & 914 & 877 & 172 & 105 & 482 & 326 & 9 & 349 \\
\hline
\end{tabular}

El Cuadro 3 expresa los parámetros de la producción de gas total y sus volúmenes fraccionales a las 8, 24 y 72 h de fermentación. Los tratamientos MP100, Z100 y M100 (M. paradisiaca, Z. mays y melaza, respectivamente) tuvieron los mayores volúmenes de gas producido $\left(544.0,467.3\right.$ y $\left.325.7 \mathrm{ml} / \mathrm{g}^{-1} \mathrm{MS}\right)$ y con diferencias $(P<0.05)$ entre los tres y también con respecto a las dietas $(P<0.05)$. Este comportamiento es propio de los alimentos con carbohidratos como los monosacáridos y almidones ${ }^{(40)}$. Por otra parte, se observó que G. sepium (G100) y L. leucocephala (L100) tuvieron un menor volumen de producción gas $(\mathrm{V})$ y fueron diferentes entre las dos arbóreas $(P<0.05)$. Al respecto, esto pudo deberse a la presencia de metabolitos secundarios (taninos) en la L. leucocephala ${ }^{(40)}$, por la presencia de altos contenidos de lignina en el follaje de ambas arbóreas $(111 \mathrm{~g} / \mathrm{kg}$ MS) o por la naturaleza fibrosa de los follajes, que disminuyen la producción de gas en comparación con dietas con mayores contenidos de carbohidratos ${ }^{(41)}$. Los tratamientos con mezclas energéticas- proteicas, aumentaron significativamente su producción de gas (V) $(P<0.05)$, observándose el efecto aditivo de los carbohidratos sobre el follaje de la L. leucocephala y G. sepium. Los valores en la tasa de producción de gas (S) fueron similares entre todos los tratamientos, a pesar de haber diferencia estadística $(P<0.05)$. En las Figuras 1 y 2 se observan los comportamientos de la PGIV por hora de los alimentos usados y dietas, respectivamente. 
Cuadro 3: Parámetros de la producción de gas total y sus volúmenes fraccionales en los alimentos y tratamientos usados en el experimento de gas in vitro en Chiapas, México

\begin{tabular}{|c|c|c|c|c|c|c|}
\hline \multirow[b]{2}{*}{ Alimentos } & \multicolumn{3}{|c|}{ Parámetros } & \multicolumn{3}{|c|}{ Volúmenes fraccionales $\left(\mathrm{ml} \mathrm{g}^{-1} \mathrm{MS}\right)$} \\
\hline & $\begin{array}{c}\mathrm{V} \\
\left(\mathrm{ml} \mathrm{g}^{-1} \mathrm{MS}\right)\end{array}$ & $\begin{array}{c}\mathrm{S} \\
\left(\mathrm{ml} \mathrm{h}^{-1}\right)\end{array}$ & $\begin{array}{c}\mathbf{L} \\
(\mathbf{h})\end{array}$ & $\mathrm{V}_{8}$ & $\mathbf{V}_{24}$ & $V_{72}$ \\
\hline P100 (control) & $266.3^{\text {de }}$ & $0.03^{\mathrm{ab}}$ & $11.2^{\mathrm{a}}$ & $15.1^{\mathrm{e}}$ & $100.5^{\mathrm{d}}$ & $159.7^{b}$ \\
\hline G100 & $253.0^{\mathrm{e}}$ & $0.03^{\mathrm{ab}}$ & $9.0^{\mathrm{b}}$ & $28.7^{\text {ed }}$ & $85.9^{\text {de }}$ & $144.8^{\mathrm{bcd}}$ \\
\hline L100 & $180.8^{\mathrm{f}}$ & $0.03^{\mathrm{ab}}$ & $2.7^{\mathrm{f}}$ & $40.6^{\mathrm{cd}}$ & $63.2^{\mathrm{e}}$ & $81.9^{\mathrm{e}}$ \\
\hline MP100 & $544.9^{\mathrm{a}}$ & $0.03^{\mathrm{ab}}$ & $3.7^{\mathrm{ef}}$ & $117.7^{\mathrm{a}}$ & $250.0^{\mathrm{a}}$ & $206.4^{\mathrm{a}}$ \\
\hline $\mathrm{Z} 100$ & $467.3^{\mathrm{b}}$ & $0.04^{\mathrm{a}}$ & $6.2^{\mathrm{c}}$ & $44.1^{\mathrm{cd}}$ & $271.2^{\mathrm{a}}$ & $194.7^{\mathrm{a}}$ \\
\hline M100 & $325.7^{\mathrm{c}}$ & $0.04^{\mathrm{a}}$ & $2.6^{\mathrm{f}}$ & $71.6^{\mathrm{b}}$ & $166.9^{\mathrm{b}}$ & $119.5^{\mathrm{d}}$ \\
\hline $\begin{array}{l}\text { Tratamientos } \\
\text { D1LM }\end{array}$ & $299.8^{\mathrm{cd}}$ & $0.03^{\mathrm{b}}$ & $4.7^{\text {cde }}$ & $51.7^{\mathrm{c}}$ & $105.0^{\mathrm{c}}$ & $149.9^{\mathrm{bc}}$ \\
\hline D2LMP & $308.9^{\mathrm{cd}}$ & $0.03^{\mathrm{ab}}$ & $5.7^{\mathrm{cd}}$ & $46.8^{c}$ & $119.7^{\mathrm{cd}}$ & $152.4^{\mathrm{bc}}$ \\
\hline D3GM & $293.6^{\text {cde }}$ & $0.03^{\mathrm{ab}}$ & $4.5^{\mathrm{de}}$ & $54.0^{c}$ & $115.6^{\mathrm{cd}}$ & $134.3^{\mathrm{bcd}}$ \\
\hline D4GMP & $337.4^{\mathrm{c}}$ & $0.03^{\mathrm{ab}}$ & $5.6^{\mathrm{cd}}$ & $52.6^{\mathrm{c}}$ & $147.1^{\mathrm{bc}}$ & $151.5^{\mathrm{cb}}$ \\
\hline D5GLMPM & $292.3^{\text {cde }}$ & $0.03^{\mathrm{ab}}$ & $3.6^{\mathrm{fe}}$ & $57.5^{\mathrm{cb}}$ & $122.2^{\mathrm{cd}}$ & $128.7^{\mathrm{cd}}$ \\
\hline
\end{tabular}

$\mathrm{V}=$ volumen máximo de producción de gas; $\mathrm{S}=$ tasa de producción constante de gas; $\mathrm{L}=$ fase Lag ó tiempo de retraso (h); $\mathrm{V}_{8=}$ volumen fraccional generado en la fracción rápida de la fermentación $(0-8 \mathrm{~h}) ; \mathrm{V}_{24}=$ volumen fraccional de gas $\left(\mathrm{ml} . \mathrm{g}^{-1}\right)$ generado en la fracción media $(8-24 \mathrm{~h}) ; \mathrm{V}_{72}=$ volumen fraccional de gas generado en la fracción lenta $(24-72 \mathrm{~h})$.

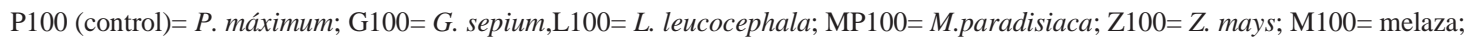
$\mathrm{D} 1 \mathrm{LM}=47 \%$ P. maximum, $30 \%$ L. Leucocephala, $8 \%$ Z. mays, $15 \%$ melaza; D2LMP $=47 \%$ P. maximum, $30 \%$ L. Leucocephala $8 \%$ Z. mays, $15 \%$ M. paradisiaca; D3GM= 47\% P. maximum, 30\% G. sepium, $8 \%$ Z. mays, $15 \%$ Melaza; $\mathrm{D} 4 \mathrm{GMP}=48 \%$ P. maximum, $30 \%$ G. sepium, $7 \%$ Z. mays, $15 \%$ M. paradisiaca; D5GLMPM=47\% P. maximum, $16 \%$ G. sepium $17 \%$ L. leucocephala $5 \%$ M. paradisiaca, $5 \%$, Z. mays, $10 \%$ melaza.

${ }^{\text {abcde }}$ Letras distintas en una misma columna indican diferencias significativas entre tratamientos $(\alpha=0.05)$.

Figura1: Comportamiento del control y de las materias primas usados en el experimento de gas In vitro en Chiapas, México

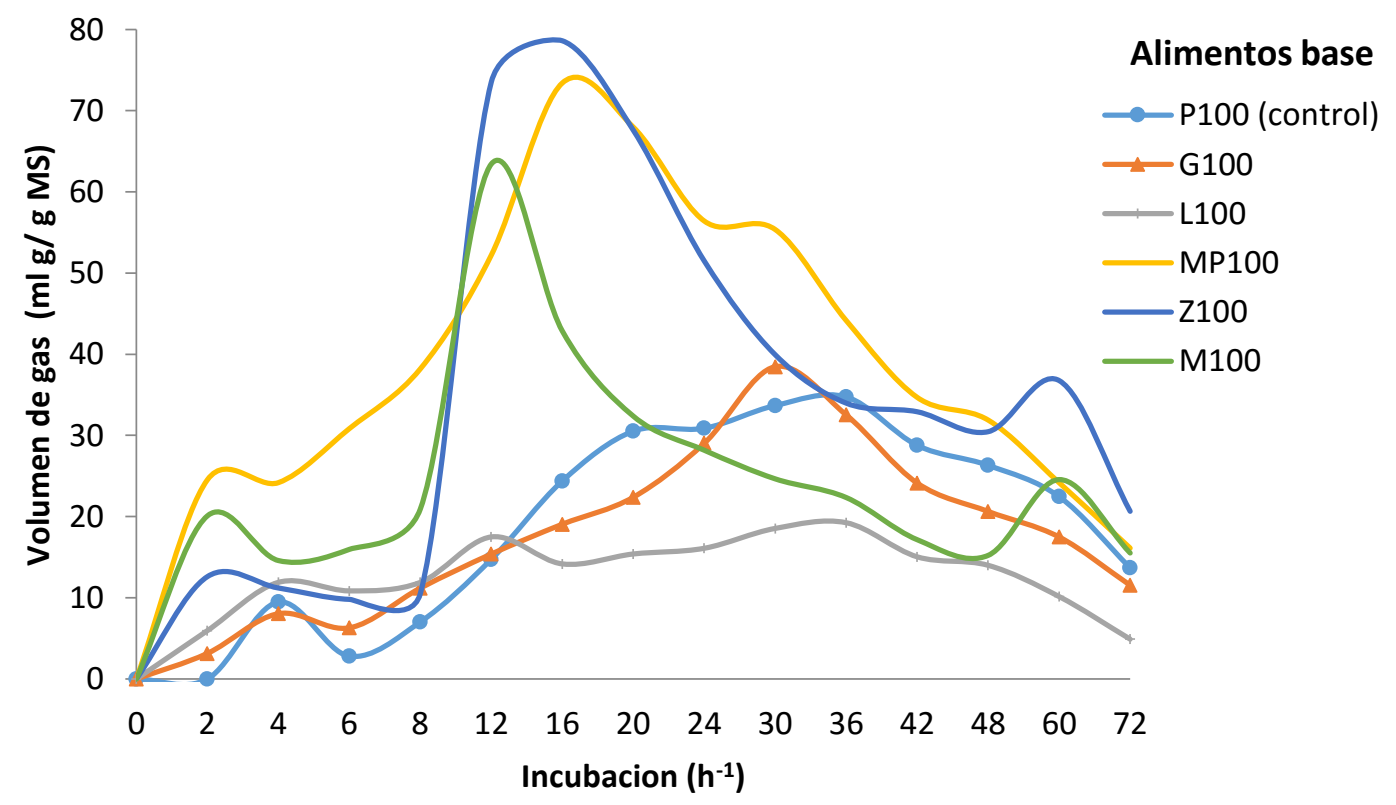

P100 (control)= Panicum máximum; G100= Gliricidia sepium, L100= Leucaena leucocephala MP100= Musa paradisiaca $; \mathrm{Z100}=$ Zea mays $; \mathrm{M100}=$ melaza de caña . 
Figura 2: Producción de gas in vitro $(\mathrm{ml}$ gas/h) de 5 dietas utilizadas en la alimentación de ovinos en un sistema silvopastoril en Chiapas México

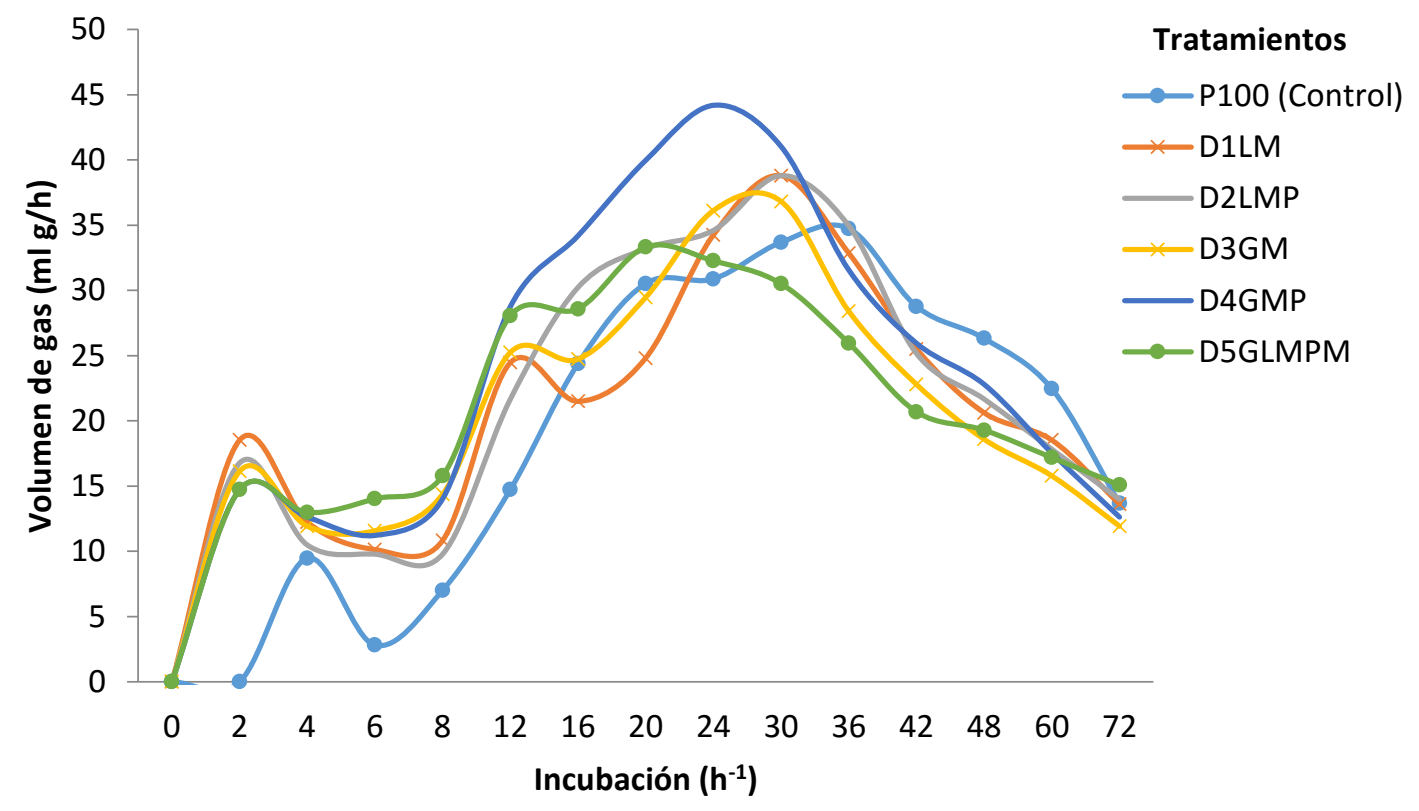

$\mathrm{P} 100($ control $)=P$. máximum $; \mathrm{D} 1 \mathrm{LM}=47 \%$ P. maximum, $30 \%$ L. Leucocephala, $8 \%$ Z. mays, $15 \%$ melaza; D2LM $=47 \%$ P. maximum, 30\% L. Leucocephala 8\% Z. mays, 15\% M. paradisiaca; D3GM: 47\% P. maximum, 30\% G. sepium, 8\% Z. mays, $15 \%$ melaza; D4GMP = 48\% P. maximum, 30\% G. sepium., 7\% Zea mays, 15\% M. paradisiaca; D5GLMPM = 47\% P. maximum, 16\% G. sepium $17 \%$ L. leucocephala. $5 \%$ M. paradisiaca., $5 \%$, Z. mays, $10 \%$ melaza.

Los alimentos como el banano (MP100) y la melaza (M100) inician su fermentación rápidamente, incrementándola durante la fase intermedia de incubación y disminuyendo rápidamente. Sin embargo, en los tratamientos que se encuentran mezclas de forrajes con fuentes energéticas, la producción de gas y tasa de fermentación es más lenta inicialmente, sin embargo en las horas intermedias de incubación, la producción de gas (V) aumenta y se mantiene por más tiempo (Figura 2). Al respecto, se sabe que durante la fermentación el sustrato se hidrata y se coloniza por los microorganismos ruínales, y dependiendo de la cantidad y tipo de carbohidratos presentes, se origina el volumen de gas y su efecto sobre la digestibilidad de la $\mathrm{MS}^{(42,43)}$.

El Cuadro 4 muestra los resultados en la DIVMS, IPEGF y producción total de $\mathrm{CH}_{4}$. Los forrajes como el P. máximum y la L. leucocephala presentaron la menor DIVMS a las 72 h (50.9 y $29.9 \%$ respectivamente) en comparación de la G. sepium y las dietas diseñadas $(P \leq 0,05)$. Resalta el valor bajo de la DIVMS de la L. leucocephala respecto a otros estudios realizados bajo condiciones in vitro e in vivo ${ }^{(34,42,43)}$. Esto pudo deberse, como ya se indicó, a que el follaje colectado de la arbórea estuvo en un estado de madurez avanzado y a su contenido alto de lignina. Las DIVMS más altas a las $24 \mathrm{~h}$ y $72 \mathrm{~h}$ fueron observadas $(P \leq 0,05)$ en los los alimentos M100 (Z. mays), Z100 (Melaza) y MP100 ( $M$. paradisiaca). Los valores intermedios de DIVMS $(P<0.05)$ se encontraron en los tratamientos-dieta D1LM, D2LMP, D3GM, D4GMP y D5GLMPM, los cuales tuvieron un incremento lineal (Figura 1), observándose en estas la contribución en la fermentación 
y digestibilidad del follaje de G. sepium y L. leucocephala. La inclusión de fuentes energéticas (tratamientos D3GM y D4GMP) permitió una mejor digestibilidad $(P \leq 0,05)$ en comparación con los tratamientos D2LMP, y P100 y L100 $(P \leq 0,05)$. Mientras que la digestibilidad encontrada para G. sepium es similar a la reportada en otro trabajo ${ }^{(43)}$. La alta digestibilidad encontrada en las fuentes energéticas (MP100, Z100 Y M100) es debido a su alto contenido de azucares solubles. En este contexto, cuando una dieta es balanceada con altos contenidos de G. sepium y melaza, la digestibilidad y aprovechamiento es mayor debido a la sincronía entre proteína y energía contenida en la $\operatorname{dieta}^{(44)}$.

Cuadro 4: $\mathrm{CH}_{4}, \mathrm{CO}_{2}$, DIVMS, IPEGF y $\mathrm{CH}_{4}$ Total, obtenidos en la fermentación de los tratamientos usados en el experimento de gas In vitro en Chiapas, México

\begin{tabular}{|c|c|c|c|c|c|c|c|}
\hline Tratamientos & $\begin{array}{l}\mathrm{CH}_{4} \\
(\%)\end{array}$ & $\begin{array}{l}\mathrm{CO}_{2} \\
(\%)\end{array}$ & $\begin{array}{c}\text { DIVMS } 24 \\
\text { h } \\
(\%) \\
\end{array}$ & $\begin{array}{c}\text { DIVMS72 } \\
4 \mathrm{~h} \\
(\%) \\
\end{array}$ & $\begin{array}{c}\text { IPEGF/MS } \\
24 \mathrm{~h}\end{array}$ & $\begin{array}{c}\text { IPEGF/MS } \\
72 \mathrm{~h}\end{array}$ & $\begin{array}{c}\mathrm{CH}_{4} \\
\text { (L CH} 4 / \mathrm{kg} \\
\text { MSDIG) } \\
\end{array}$ \\
\hline P100 (control) & $22.5^{\text {bcd }}$ & $77.5^{\mathrm{abc}}$ & $33.7^{\mathrm{f}}$ & $50.9^{\mathrm{e}}$ & $791.0^{\mathrm{a}}$ & $523.5^{\mathrm{cd}}$ & $1.55^{\mathrm{d}}$ \\
\hline G100 & $23.2^{\mathrm{bcd}}$ & $76.8^{\mathrm{abc}}$ & $51.0^{\mathrm{cd}}$ & $60.1^{\mathrm{cd}}$ & $496.8^{\mathrm{e}}$ & $420.9^{\mathrm{e}}$ & $1.68^{\mathrm{d}}$ \\
\hline L100 & $30.8^{\mathrm{a}, \mathrm{b}}$ & $69.2^{\mathrm{cd}}$ & $28.8^{\mathrm{f}}$ & $29.9^{\mathrm{f}}$ & $628.1^{\mathrm{bcd}}$ & $606.6^{\mathrm{ab}}$ & $1.94^{\mathrm{d}}$ \\
\hline MP100 & $18.1^{\mathrm{d}}$ & $81.9^{\mathrm{a}}$ & $77.0^{\mathrm{b}}$ & $83.6^{\mathrm{b}}$ & $708.1^{\mathrm{ab}}$ & $652.1^{\mathrm{a}}$ & $15.75^{\mathrm{b}}$ \\
\hline Z100 & $16.4^{\mathrm{d}}$ & $83.6^{\mathrm{a}}$ & $80.1^{\mathrm{b}}$ & $87.0^{\mathrm{b}}$ & $583.6^{\mathrm{cde}}$ & $537.2^{\mathrm{cd}}$ & $28.59^{\mathrm{a}}$ \\
\hline M100 & $17.9^{\mathrm{d}}$ & $82.1^{\mathrm{a}}$ & $92.7^{\mathrm{a}}$ & $92.4^{\mathrm{a}}$ & $351.4^{\mathrm{f}}$ & $352.5^{\mathrm{f}}$ & $9.03^{c}$ \\
\hline D1LM & $31.9^{\mathrm{a}}$ & $68.1^{\mathrm{d}}$ & $44.4^{\mathrm{e}}$ & $56.6^{\mathrm{d}}$ & $678.6^{\mathrm{bc}}$ & $529.7^{\mathrm{cd}}$ & $8.82^{\mathrm{c}}$ \\
\hline D2LMP & $27.0^{\mathrm{abc}}$ & $73.0^{\mathrm{bcd}}$ & $44.9^{\mathrm{de}}$ & $50.9^{\mathrm{e}}$ & $690.0^{\mathrm{b}}$ & $606.5^{\mathrm{ab}}$ & $8.83^{\mathrm{c}}$ \\
\hline D3GM & $24.2^{\mathrm{abcd}}$ & $75.8^{\mathrm{abcd}}$ & $55.1^{\mathrm{c}}$ & $61.9^{c}$ & $533.0^{\mathrm{de}}$ & $474.2^{\mathrm{de}}$ & $6.32^{\mathrm{cd}}$ \\
\hline D4GMP & $21.9^{\mathrm{cd}}$ & $78.1^{\mathrm{ab}}$ & $54.5^{\mathrm{c}}$ & $61.1^{\mathrm{cd}}$ & $619.4^{\mathrm{bcd}}$ & $552.1^{\mathrm{bc}}$ & $9.60^{c}$ \\
\hline D5GLMPM & $22.3^{\mathrm{bcd}}$ & $77.7^{\mathrm{abc}}$ & $51.7^{\mathrm{c}}$ & $56.6^{\mathrm{d}}$ & $565.5^{\mathrm{de}}$ & $516.9^{\mathrm{cd}}$ & $6.31^{\mathrm{cd}}$ \\
\hline
\end{tabular}

$\mathrm{CH}_{4}=$ metano + gases menores in vitro; $\mathrm{CO}_{2}=$ bióxido de carbono in vitro, DIVMS24= digestibilidad in vitro de la materia seca a $24 \mathrm{~h}$, DIVMS72= digestibilidad in vitro de la materia seca a $72 \mathrm{~h}$;

IPEGF/MS24= índice potencial de emisión de gas fermentable a 24 h de fermentación; IPEGF/MS72= índice potencial de emisión de gas fermentable a 72 h de fermentación; $\mathrm{CH}_{4}=$ concentración de metano en $24 \mathrm{~h}$ de incubación.

$\mathrm{P} 100($ control $)=$ P. máximum $; \mathrm{G} 100=$ G. sepium $; \mathrm{L} 100=$ L. leucocephala $; \mathrm{MP} 100=$ M.paradisiaca $; \mathrm{Z} 100=$ Z. mays; M100= melaza; D1LM=47\% P. maximum, 30\% L. Leucocephala, 8\% Z. mays, 15\% melaza; $\mathrm{D} 2 \mathrm{LMP}=47 \%$ P. maximum, 30\% L. Leucocephala 8\% Z. mays, $15 \%$ M. paradisiaca; D3GM: $47 \%$ P. maximum, $30 \%$ G. sepium, $8 \%$ Z. mays, $15 \%$ melaza; D4GMP= 48\% P. maximum, $30 \%$ G. sepium, $7 \%$ Z. mays, $15 \%$ M. paradisiaca; D5GLMPM=47\% P. maximum, $16 \%$ G. sepium $17 \%$ L. leucocephala $5 \%$ M. paradisiaca, $5 \%$, Z. mays, $10 \%$ melaza.

Letras distintas en una misma columna indican diferencia estadísticamente significativas entre los tratamientos $(\alpha=0.05)$.

La máxima producción total de $\mathrm{CH}_{4}$ (L/Kg MSDG) fue observada en el maíz (Z100) y banano (MP100) $(P \leq 0,05)$. Los valores más bajos se obtuvieron con el control (P100), G. sepium (G100) y L. leucocephala (L100), no habiendo diferencias $(P>0.05)$ entre ellos. Los tratamientos que tuvieron mezclas de los alimentos usados, como D5GLMPM, D3GM, D1LM, D2LMP y D4GMP, presentaron valores intermedios $(P>0.05)$. De los tratamientos con dietas mixtas de energía y proteína, la dieta D5GLMPM tuvo el menor 
valor de producción de $\mathrm{CH}_{4}$, mostrando la importancia de la inclusión de forrajes asociada entre carbohidratos ${ }^{(45,46)}$. Estos autores resaltan que el tipo de carbohidratos determina la taza de pasaje, afectando la producción de $\mathrm{CH}_{4}$ por gramo de sustrato digerido. El tipo de carbohidrato parece ser un factor determinante en la producción de $\mathrm{CH}_{4}{ }^{(47)}$; ya que puede estar mediada por una menor disponibilidad de carbohidratos digeribles ${ }^{(48)}$. También una concentración de $550 \mathrm{~g} \mathrm{~kg}^{-1} \mathrm{MS}$, se encuentra por arriba de la concentración que afecta negativamente el consumo voluntario y la digestibilidad de los nutrientes en los animales ${ }^{(49)}$. Adicionalmente los follajes de arbóreas y arbustivas contienen baja concentración de fracciones estructurales ${ }^{(44)}$ haciéndolos más susceptibles a la degradación y acción de las bacterias, provocando un aumento de la tasa de pasaje disminuyendo la producción de gas total y por lo tanto una menor producción de $\mathrm{CH}_{4}$ entérico $^{(36,50)}$.

La cuantificación de GEI provenientes de la fermentación ruminal y el diseño de índices para evaluar el potencial de contaminación ambiental y diseñar estrategias sustentables de manejo animal, ha sido de interés por agencias de investigación y desarrollo ${ }^{(51,52)}$. El Cuadro 4, muestra que hay una amplia variación $(P<0.001)$ en los IPEGF/MS, tanto a las $24 \mathrm{~h}$ como $72 \mathrm{~h}$ en los alimentos y tratamientos evaluados. Destaca observar, que los índices más bajos corresponden a M100 y G100 (496. 8 y 420.9 ml.g-1/DIVMS a 24 y 72 h). Los tratamientos con mayor IPEGF fueron MP100, con 708.1 y $652.1 \mathrm{ml} . \mathrm{g}^{-1} / \mathrm{DIVMS}$ a 24 y 72 h respectivamente. De los tratamientos que incluyen follaje de arbóreas y fuentes energéticas, el menor índice correspondió a la mezcla D3GM. Los datos encontrados sugieren la importancia del tipo de follaje proveniente de árboles forrajeros en asociación con el tipo de carbohidrato, especialmente si este último tiene proceso lento de fermentación, como son los almidones ${ }^{(53)}$.

\section{Conclusiones e implicaciones}

Este estudio sugiere que en sistemas silvopastoriles, la combinación del follaje de arbóreas forrajeras con fuentes energéticas locales, especialmente la melaza y bananos, pueden mejorar el valor nutritivo de los forrajes para permitir una mejor respuesta en la producción animal y en la mitigación de gases de efecto invernadero (GEI), como el metano. Así, la combinación de $30 \%$ en MS de follaje de arbóreas como G. sepium y L. leucocephala con fuentes energéticas locales como melaza y banano, contribuir a tener sistemas ganaderos de bajas emisiones de $\mathrm{CH}_{4}$. Por su mejoramiento en la calidad de las dietas para sistemas silvopastoriles y en contribuir en una baja de emisiones de $\mathrm{CH}_{4}$, se sugiere el manejo de arbóreas forrajeras como G. sepium y L. leucocephala, incorporando fuentes energéticas locales. Importante en un futuro hacer estudios de respuesta animal (ganancias de peso) y balance bio-económico, para encontrar viabilidad económicasocial en la adaptación de estas estrategias en un contexto de mejorar la producción animal, contribuir en el bienestar social de los productores y la mitigación de GEI. 


\section{Agradecimientos}

Se agradece el financiamiento para la investigación bajo el proyecto "Cuantificación de emisiones de metano entérico y óxido nitroso en ganadería bovina en pastoreo y diseño de estrategias para la mitigación en el sureste de México" (SEP-CONACYT CB 2014-1 No. 242541).

\section{Literatura citada:}

1. FAO. Food and Agriculture: Key to Achieving in the 2030. Agenda for Sustainable Development. FAO, Rome. 2016.

2. IPCC. Climate Change 2014: Impacts, adaptation, and vulnerability report of the Intergovernmental Panel on Climate Change. Field C, et al. editors. Cambridge, UK 2014.

3. O`Mara FP. The significance of livestock in global greenhouse gas emissions today and in the near future. Anim Feed Sci Technol 2011;(166-167):7-15.

4. Ibrahim M, Chacón M, Cuartas C, Naranjo J, Ponce G, Vega P, Casasola F, Rojas J. Almacenamiento de carbono en el suelo y la biomasa arbórea en sistemas de usos de la tierra en paisajes ganaderos de Colombia, Costa Rica y Nicaragua. Agroforestería las Américas 2007;(45):27-36.

5. Avila-Foucalt V, Revollo FD. Análisis financiero y percepción de los servicios ambientales de un sistema silvopastoril: un estudio de caso en los Tuxtlas, México. Rev Iber Econ Ecol 2014;(21):17-33.

6. Solorio S, Wright M, Franco M, Basu S, Sarabia S, Ramirez L, et al. Silvopastoral systems: Best agroecological practice for resilient production systems under dryland and drought conditons. Ahmed M, Stockle C. editors. Quantificaction of climate variability, adaptation and mitigation for agricultural sutainability. Australis: Springer; 2002:233-250.

7. Murgueitio E, Chará J, Barahona R, Cuartas C, Naranjo J. Los Sistemas Silvopastoriles Intensivos (SSPI), herramienta de mitigación y adaptación al cambio climático. Trop Subtrop Agroecosystems 2014;(17):501-507.

8. Palmer L. A new climate for grazing livestock. Nat Clim Chang 2014;(4):321-323. 
9. Ibrahim M, Villanueva C, Casasola F. Sistemas silvopastoriles como una herramienta para el mejoramiento de la productividad y rehabilitación ecológica de paisajes ganaderos en Centro América. Agroforesteria las Américas 2007;(15):1-34.

10. Kass ML. Determinación del nitrógeno en los alimentos. Ruiz, M, Ruiz A. editores. Nutrición de rumiantes, Guía Metodológica de Investigación. Costa Rica, ALPARISPAL 1990:49-58.

11. Sirohi SK. Mitigation options for enteric methane emissions from dairy animals: an evaluation for potential projects in India. Mitig Adapt Strateg Glob Chang 2007;(12):259-274.

12. Piñeiro-Vázquez A, Ayala-Burgos A, Chay-Canul A, Ku-Vera J. Dry matter intake and digestibility of rations replacing concentrates with graded levels of Enterolobium cyclocarpum in Pelibuey lambs. Trop Anim Health Prod 2013;(45):577-583.

13. Jiménez-Ferrer G, Mendoza-Martinez G, Soto-Pinto L, Alayón-Gamboa A. Evaluation of local energy sources in milk production in a tropical silvopastoral system with Erythrina poeppigiana. Trop Anim Health Prod 2015;(47):903-908.

14. Norton B. Anti-nutritive and toxic factors in forage tree legumes. Gutteridge R, Shelton $\mathrm{H}$ editors. Forage tree legumes in tropical agriculture. UK: Cab International; 1993:202-215.

15. Piñeiro-Vázquez A, Canul-Solís J, Alayón-Gamboa J, Chay-Canul A, Ayala-Burgos A, Aguilar-Pérez C, et al. Potential of condensed tannins for the reduction of emissions of enteric methane and their effect on ruminant productivity. Arch Med Vet 2015;(47):263-272.

16. Yáñez-Ruiz D, Hart K, Martin-Garcia A, Ramos S, Newbold C. Diet composition at weaning affects the rumen microbial population and methane emissions by lambs. Aust J Exp Agric 2008;(48):186-188.

17. Min B, Solaiman S, Shange R, Eun J. Gastrointestinal bacterial and methanogenic Archaea diversity dynamics associated with condensed tannin-containing pine bark diet in goats using 16S rDNA amplicon pyrosequencing. Int J Microbiol 2014; Article ID 141909, 11 pages. doi.org/10.1155/2014/141909

18. Herrera-López M, Rivera-Lorca J, Ortega-Reyes L, Escobedo-Mex J, Magaña M, Sanginés-García J, Sierra-Vázquez Á, Contenido nutritivo y factores antinutricionales de plantas nativas forrajeras del norte de Quintana Roo. Tec Pecu Mex 2008;(46):205-215.

19. Puchala R, Animut G, Patra A K, Detweiler G D, Wells J E, Varel V H, Sahlu T, Goetsch A. Methane emissions by goats consuming Sericea lespedeza at different feeding frequencies. Anim Feed Sci Technol 2012;(175):76-84. 
20. Soltan YA, Morsy AS, Lucas RC, Abdalla AL. Potential of mimosine of Leucaena leucocephala for modulating ruminal nutrient degradability and methanogenesis. Anim Feed Sci Technol 2017; (223):30-41.

21. Pérez Esaú, Soca Mildrey, Díaz L, Corzo M. Comportamiento etológico de bovinos en sistemas silvopastoriles en Chiapas, Pastos y Forrajes 2008;(31):161-172.

22. Van-Soest, P, Robertson J, Lewis B. Methods for dietary fiber, neutral detergent fiber, and non-starch polysaccharides in relation to animal nutrition, J Dairy Sci 1991;74:3583-3597.

23. AOAC. Official Methods for Analysis. Association of Official Analysis Chemist, Gaitersburgh, MD, USA. 2006.

24. Price ML, Van SC, Butler L. A critical evaluation of the vanillin reaction as an assay for tannin in sorghum grain, J Agric Food Chem, 1978;26(5):1214-1218. doi: 10.1021/jf60219a031

25. Theodorou M K, Williams BA, Dhanoa M S, McAllan AB, France J. A simple gas production method using a pressure transducer to determine the fermentation kinetics of ruminant feeds. Anim Feed Sci Technol 1994;(48):185-197.

26. Williams, BA. Cumulative gas-production techniques for forage evaluation. In: Forage evaluation in ruminant nutrition. Givens, D et al., editors. UK : CABI, Pub; 2000:189-213.

27. Alexander RH, McGown M. The routine determination of in vitro digestibility of organic matter in forages-an investigation of the problems associated with continuos large-scale operation. J Brit Grass Soc 1966;(21):140-147.

28. Blummel M, Orskov ER. Comparison of in vitro gas production and nylon bag degradability of roughages in predicting food intake in cattle. Anim Feed Sci Technol 1993;(40):109-119.

29. Miranda LA, Vázquez MP, Améndola MR, Sandoval GL, González OR. Cuantificación de las fracciones fermentables de alfalfa y tuna por la técnica de producción de gas [resumen]. Congreso de la Asociación Latinoamericana de Producción Animal. Puerto Varas, Chile. 2015:575.

30. Menke K, Steingass H. Estimation of the energetic feed value obtained from chemical analysis and in vitro gas production using rumen fluid. Anim Res Dev $1988 ;(28): 7-55$.

31. Cáceres LM. Índice de contaminación atmosférica y productividad de vacas Jersey y sus cruzas con Holstein en pastoreo, [tesis maestría]. Texcoco, México. Universidad Autónoma Chapingo; 2016. 
32. Bartha R, Pramer D. Features of flask and method for measuring the persistence and biological effects of pesticides in soil. Soil Sci. 1965; 100; (1): 68-70

33. Cochran WG, Cox GM. Diseños experimentales. 2a ed. México: Trillas; 1991.

34. SAS. User's Guide SAS/STAT. Sas Institute, Cary, NC, USA. 2015.

35. Abdulrazak S, Muinga R, Thorpe W, Ørskov ER. Supplementation with Gliricidia sepium and Leucaena leucocephala on voluntary food intake, digestibility, rumen fermentation and live weight of crossbred steers offered Zea mays stover. Livest Prod Sci 1997;(49):53-62.

36. Morgavi D P, Archimède $\mathrm{H}$, Marie-Magdeleine $\mathrm{C}$, Popova $\mathrm{M}$, Bousseboua $\mathrm{H}$, Doreau M. Potential of tannin-rich plants for modulating ruminal microbes and ruminal fermentation in sheep. J Anim Sci 2015;(93):334-347.

37. Borel, R. Nutrición de rumiantes: Aspectos críticos de las metodologías de evaluación nutritiva de árboles y arbustos forrajeros. Guía metodológica de investigación. ALPA-IICA, Costa Rica 1990:21-31.

38. Piñeiro-Vázquez AT, Jiménez-Ferrer G, Chay-Canul AJ, Casanova-Lugo F, DíazEcheverría VF, Ayala-Burgos A, et al. Intake, digestibility, nitrogen balance and energy utilization in heifers fed low-quality forage and Leucaena leucocephala. Anim Feed Sci Technol 2017. Vol. 228, pag. 194-201. doi.org/10.1016/j.anifeedsci. 2017.04.009

39. Van Soest PJ. Development of a comprehensive system of feed analyses and its application to forages. J Anim Sci 1967;(26):119-128.

40. Van Soest PJ. Nutritional ecology of the ruminant. Corvallis, Oregon: O\&B Books Inc; 1982.

41. Cárdenas J, Sandoval C, Solorio F. Composición química de ensilajes mixtos de gramíneas y especies arbóreas de Yucatán. Tec Pecu Méx 2003;(41):283-294.

42. Juárez F, Contreras J, Montero M. Tasa de cambios con relación a edad en rendimiento, composición química y digestibilidad de cinco pastos tropicales. Decima Cuarta Reun Cient Tecnol Forest Agropecu. Veracruz, Mex. 2001.

43. Williams BA. Cumulative gas-production technique for forage evaluation. Eds: Givens D, et al. editors. Forage Evaluation in Ruminant Nutrition, UK 2000; 189 213.

44. Reed J. Nutritional toxicology of tannins and related polyphenols in forage legumes. J Anim Sci 1995;(73):1516-1528.

45. Molina-Botero C, Cantet J, Montoya S, Correa-Londoño G, Barahona-Rosales R. In vitro methane production from two tropical grasses alone or in combination with 
Leucaena leucocephala or Gliricidia sepium. CES Med Vet y Zootec 2013;(8):1531.

46. Archiméde H, Rira M, Barde D, Labirin F, Marie-Magdeleine C, et al. Potential of tannin-rich plants, Leucaena leucocephala, Glyricidia sepium and Manihot esculenta, to reduce enteric methane emissions in sheep. J Anim Physiol Anim Nutr 2016;(100):1149-1158.

47. Fernández-Mayer A. Efecto de la sincronización de energía-proteína sobre la performance animal. EEA INTA Bordenave 2001:7-13.

48. Eugene M, Archimèede H, Sauvant, D. Quantitative metanalysis on the effects of defaunation of the rumen on growth, intake and digestion in ruminants, Livestock Prod Sci 2004;85:81-97.

49. Van Soest PJ. Symposium on factors influencing the voluntary intake of herbage by ruminants: voluntary intake in relation to chemical composition and digestibility. $\mathrm{J}$ Anim Sci1965: 4(3):834-843.

50. Animut G, Puchala R, Goetsch AL, Patra AK, Sahlu T, Varel VH, Wells J. Methane emission by goats consuming diets with different levels of condensed tannins from lespedeza. Anim Feed Sci Technol 2008;(144):212-227.

51. Johnson KA, Johnson DE. Methane emissions from cattle. J Anim Sci 1995;73 (8):2483-2492.

52. McAllister TA, Cheng KJ, Okine EK y Mathison GW. Dietary, environmental and microbiological aspects of methane production in ruminants. Can J Anim Sci 1996;76(2):231-243.

53. Nunes L, Salomon S, Castro L, Robson E, Abdalla A. Chemical composition, degradability and methane emission potential of banana crop residues for ruminants. Trop Subtrop Agroecosyst 2014;(17):197-206. 